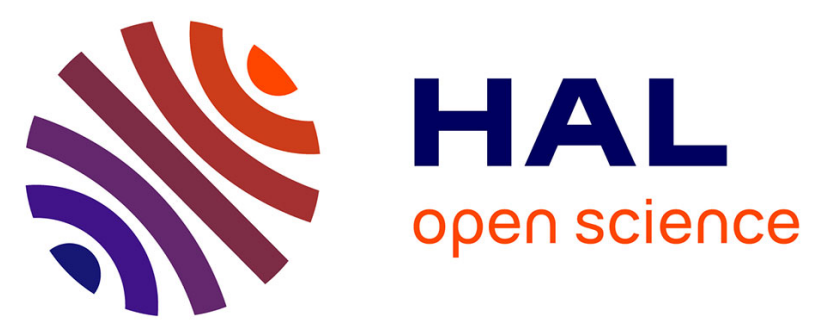

\title{
The Physics of Lipid Droplet Nucleation, Growth and Budding
}

Abdou Rachid Thiam, Lionel Forêt

\section{To cite this version:}

Abdou Rachid Thiam, Lionel Forêt. The Physics of Lipid Droplet Nucleation, Growth and Budding. Biochimica et Biophysica Acta Molecular and Cell Biology of Lipids, 2016, 1891 (8, Part A), pp.715722. 10.1016/j.bbalip.2016.04.018 . hal-01311683

\section{HAL Id: hal-01311683 https://hal.sorbonne-universite.fr/hal-01311683}

Submitted on 4 May 2016

HAL is a multi-disciplinary open access archive for the deposit and dissemination of scientific research documents, whether they are published or not. The documents may come from teaching and research institutions in France or abroad, or from public or private research centers.
L'archive ouverte pluridisciplinaire $\mathbf{H A L}$, est destinée au dépôt et à la diffusion de documents scientifiques de niveau recherche, publiés ou non, émanant des établissements d'enseignement et de recherche français ou étrangers, des laboratoires publics ou privés. 


\title{
The Physics of Lipid Droplet Nucleation, Growth and Budding
}

\author{
Abdou Rachid Thiam ${ }^{1, *}$, Lionel Forêt ${ }^{1}$ \\ 1'Laboratoire de Physique Statistique, École Normale Supérieure, PSL Research University; \\ Université Paris Diderot Sorbonne Paris-Cité; Sorbonne Universités UPMC Univ Paris 06; CNRS; \\ 24 rue Lhomond, 75005 Paris, France.
}

*Corresponding author, thiam@lps.ens.fr

\begin{abstract}
:
Lipid droplets (LDs) are intracellular oil-in-water emulsion droplets, covered by a phospholipid monolayer and mainly present in the cytosol. Despite their important role in cellular metabolism and growing number of newly identified functions, LD formation mechanism from the endoplasmic reticulum remains poorly understood. To form a $L D$, the oil molecules synthesized in the ER accumulate between the monolayer leaflets and induce deformation of the membrane. This formation process works through three steps: nucleation, growth and budding, exactly as in phase separation and dewetting phenomena. These steps involve sequential biophysical membrane remodeling mechanisms for which we present basic tools of statistical physics, membrane biophysics, and soft matter science underlying them. We aim to highlight relevant factors that could control LD formation size, site and number through this physics description. An emphasis will be given to a currently underestimated contribution of the molecular interactions between lipids to favor an energetically costless mechanism of LD formation.
\end{abstract}

\section{Highlights}

- Lipid droplet biogenesis occurs in three steps: nucleation, growth and budding

- Phase separation and dewetting phenomena are determinant for lipid droplet formation

- Interactions between lipids allow for spontaneous lipid droplet nucleation and budding

\section{Introduction}

Lipid droplets (LDs) are dynamic organelles found in almost all organisms. They have an oil core, consisting of triglycerides (TG) and sterol esters (SE), surrounded by a phospholipid (PL) monolayer containing proteins[1-3]. From the physical chemistry standpoint, LDs represent an inverted emulsion, meaning a solution of oil-in-water droplets[4]. Despite an important role in energy metabolism and cellular homeostasis[1], and an expanding list of newly identified functions[5], the mechanism of LD formation remains largely unknown[3, 6-10]. Under nutrient rich conditions, excess metabolites are transformed mainly into TG and SE molecules, depending on 
cell type, and encapsulated between the endoplasmic reticulum (ER) monolayer leaflets[7, 9] (for convenience, we will refer to TG as the group of oil molecules forming LDs). TG molecules then gather in the ER bilayer to form an oil lens that ultimately buds off, mainly towards the cytoplasmic side. The mechanisms of nascent LD advent and growth, and its directional budding preference, are still unclear[7, 8]. Proteins certainly affect these different steps of LD formation, but the lipid chemistry, PLs and oil molecules, play an important role that is still not resolved[4, 11, 12]. The relative contribution of proteins and lipids and their coordination for LD budding are also not fully understood[7]. This review presents a thermodynamics and mechanics view of LD formation[1315], essentially based on statistical and soft matter physics. This physics standpoint offers a better understanding of LD nucleation, growth, and budding, and protein recruitment to forming LDs, and finally of potential different LD formation mechanisms in the cell.

The following steps of LD biogenesis will be discussed and analyzed: (i) nucleation, i.e. the initial accumulation of oil molecules at places in the ER membrane where they form nascent LDs, (ii) growth, i.e. size expansion, not due to synthesized TG absorption, but by ripening and fusion, and (iii) budding, i.e. the deformation of the ER monolayers leading to the appearance of a nearly spherical oil-filled protrusion. We will emphasize the role in these steps of the molecular interactions between the oil molecules and PLs constituting the ER, a contribution for LD formation that has not yet been considered.

\section{Nucleation and formation sites of LDs}

Individual TG molecules made in the ER by synthesis enzymes[16-20] are encapsulated between the ER monolayers. The first step in the LD formation process is the accumulation of the molecules to form an oil lens in the bilayer[2, 6, 7], Fig. 1. What is the mechanism responsible for the oil lens nucleation or nascent LD appearance? Where do the oil lenses form and how are they regulated? These are important questions for LD biology[21] which can be better addressed by understanding the physics of phase separation and nucleation phenomena.

\section{1) Nucleation by phase separation}

The initial nucleation of a TG lens between the two monolayers of the ER membrane could result from a simple phase separation or demixing, a well understood phenomenon in physical chemistry[22]. Individual TG molecules dispersed in a PL bilayer can condense to form droplets in order to globally reduce their interaction with PLs or proteins of the membrane, Fig. 1, exactly in the same way as oil or gas molecules in water condense into droplets or bubbles (e.g. in sparkling 
water), to minimize the contact between foreign molecules[22]. The (free) energy cost of having a TG molecule in the bilayer instead of in a forming lens is

$\Delta \mu=\varepsilon_{f}-\varepsilon_{L D}+k T \ln \phi$

where $\varepsilon_{f}$ is the energy cost for the interaction of a free TG molecule with other ER components (e.g. PLs), $\varepsilon_{L D}$ is the interaction energy between TG molecules in the lens, and the last term of (1) arises from the mixing entropy, i.e. the propensity of entropy to disperse TG in the bilayer, with $\phi$ the concentration of free TGs in the bilayer, and $k T$ the thermal energy. Spontaneous demixing of TG molecules to form nascent LDs occurs if the energy cost for dispersing TG in the bilayer is positive, i.e. $\Delta \mu>0$. This condition requires that $\varepsilon_{f}>\varepsilon_{L D}$, which means that the energy cost for the interaction between a TG and a PL molecule is larger than the one between two TG molecules; this is in general the case, as the mixing of different molecules is often energetically costly. Only when this condition is fulfilled, demixing occurs if the TG concentration in the bilayer exceeds a critical value $\phi^{*}$. The existence of a critical demixing concentration (CDC $\left.=\phi^{*}\right)$ is common to all phase-separating systems - in the context of detergency it is called the Critical Micellar Concentration (CMC)[23, 24]. A TG demixing phenomenon based on energy minimization is supported by in silico studies showing the spontaneous appearance of TG blisters in a bilayer of POPC (1-palmitoyl-2-oleoyl-sn-glycero-3-phosphocholine) above a CDC of around 3\% to the POPC concentration[25].

In the demixing scenario, the interaction of PLs with TG is crucial (table 1) for the packaging of TG into nascent LDs[11, 12, 26, 27]. For example, by inducing squalene accumulation instead of TG, demixing could be inhibited to an extent that LD formation is impeded[28]; SE and TG could also demix differently, potentially explaining the packaging of SE and TG into different LDs within the same cell[26]. Inversely, changing the PL composition could change the CDC to inhibit demixing and nucleation. Such contribution of PLs is consistent with the systematic variation of LD size and number upon PL modulation[4, 11, 12, 29-31].

\section{2) Nucleation sites}

Assuming TG demixing induces LD nucleation, basic features of nucleation phenomena presented in box 1 should also describe LD nucleation. After synthesis, individual TG molecules freely diffuse in the ER bilayer, randomly meet and assemble to form transient pre-droplet structures. Most of these structures will be short-lived (a priori much less than a millisecond[25]) and only a very small fraction of them will cross the nucleation barrier to form stable TG lenses (nascent LDs). The 
nucleation rate of the lenses depends on the height of the nucleation barrier[23, 24]; it is determined by the relative importance of the physical interactions listed in table 1, in particular the molecular interactions between PL and TG, which are a priori predominant.

On a uniform bilayer, LD nucleation would occur at random places with no specific sites. The lower the nucleation barrier, the higher the number of nucleated LDs. Yet, because of the complex morphology of the ER and the possible heterogeneities of its biochemical composition, favored sites where nucleation is energetically cheaper may exist, as will be discussed in the next section and in Fig. 2. Indeed, owing to the exponential dependency of the nucleation barrier rate with the energy barrier, box $\mathbf{1}$, the probability to nucleate LDs is considerably increased at ER sites where the nucleation energy is lowered, even slightly. In Cos-1 cells for example, these favorite sites could coincide with the ER tubular network at the cell periphery where the first generation of LDs appears there upon oleate loading[32].

\section{3) Regulation of nucleation sites}

Physical mechanisms that can locally lower the nucleation barrier and regulate nucleation sites are sketched in Fig.2. The first mechanism (Fig.2A) is related to the ER morphology. The unzipping and deformation of the ER monolayers could be energetically cheaper at places of particular topology such as protrusion tips; they are highly bent and display hydrophobic defects - reflected by PL packing defects - which are energetically favorable for TG accumulation. Three other potential mechanisms (Fig.2B-D) can be assigned the heterogeneity of the ER membrane composition. The simplest case (Fig.2B) is the accumulation of TG molecules at the places they are made by diacylglycerol O-acyltransferase 1 or 2 enzymes (DGAT1 and 2). This accumulation can occur if the lateral diffusion of TG molecules (a priori similar to PLs, ca. $1 \mu \mathrm{m}^{2} / \mathrm{sec}$ ) is slow compared to their synthesis rate; the TG concentration will increase around the synthesis sites and quickly reach the CDC to nucleate a LD. In the opposite case, if TG molecules diffused faster, they could reach sites rich in proteins important for LD formation, such as fat storage-inducing transmembrane proteins (FIT), Seipin, Perilipin 1 or 2, long-chain acyl-coenzyme A synthetase (ACSL) [7, 11, 33-36]. These proteins could favor LD nucleation by lowering the membrane bending energy (Fig.2C) or the interfacial energy between TG and the ER monolayers (Fig.2D); they could achieve this action by locally modulating the ER physical chemistry, for example, by interacting with diacylglycerols required for making TG[37], or favoring the local recruitment of specific PLs[31, 38, 39]. 
In the case LD nucleation does not require the mechanical action of proteins (random nucleation, nucleation induced by membrane topology, or nucleation near TG synthesis sites), nucleation may itself induce local changes in membrane curvature[13], thickness, and hydrophobicity, which could be sensed by proteins to bind nascent LDs[7, 32], Fig.3. Many proteins, including amphipathic helix-binding proteins, found on LDs, have indeed the ability to sense and induce local spontaneous curvatures in membranes[13, 40, 41]. Whether the spontaneous TG demixing mediates protein recruitment to nascent LDs[7, 42], or, inversely, proteins determine nascent LD nucleation sites, remains unknown. Both could happen in the ER[42], and their dual occurrence could explain how cells achieve LDs of different biological functions[6, 7,43$]$. This is an important question of LD biology.

\section{Box 1: Basics of nucleation phenomena Insert here Fig.box1}

Nucleation of droplets in a fluid mixture undergoing phase separation is a stochastic process. Individual molecules randomly diffuse in their environment, occasionally come into contact and assemble to form transient small pre-droplet structures[22-24]. The large majority of these predroplets rapidly re-disperse and only a very small fraction of them survives and keeps growing. This nucleation barrier feature is the consequence of the characteristic "bell" shape of the droplet (free) energy, function of the droplet volume, Fig.box1. The maximum of this energy is reached at

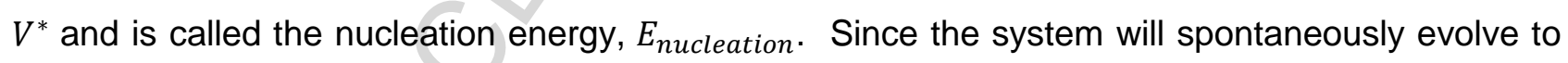
decrease the free energy, large pre-droplets $\left(V>V^{*}\right)$ will grow while small pre-droplets $\left(V<V^{*}\right)$ will collapse[22]. With the help of the molecular (thermal) agitation, some pre-droplets can nevertheless "climb the hill" and reach $V^{*}$, from which they will keep growing by absorbing free molecules diffusing around. The nucleation rate goes as $\sim \exp \left(-E_{\text {nucleation }} / k T\right):$ the higher the nucleation energy barrier, the less probable is the nucleation of a new droplet.

The bell shape of $E(V)$, and hence the existence of a nucleation energy, results from the competition of two contributions.

$E(V)=-\Delta \mu \times \rho V+E_{S}(V)$

The parameter $\Delta \mu$ reflects the energy gain of a free molecule absorbed by a droplet; it is given by equation (1) in the case of LDs and dependent on TG-TG and TG-PL interactions; $\rho$ is the density 
of molecules. The term $\Delta \mu \times \rho V$ of $\mathrm{E}$ promotes droplet growth and dominates when $V>V^{*}$. The second term $E_{S}(\mathrm{~V})$ is an energy cost due to the droplet interface and mainly depends on TG-PL interaction in the case of forming LDs. This term opposes droplet formation and dominates when $V<V^{*}$. In the case of LDs, additional effects should contribute to $E_{s}$ : the unzipping of the bilayer and the deformation of the monolayers surrounding the droplet, table 1.

\section{Communication between formation sites for LD growth}

From cell to cell, LD number and size vary. The biological relevance of this diversity remains unclear[43, 44]. For different cells, this variation may originate from the communication between LD nucleation sites, reducing the number of formed LDs.

TG molecules made in the ER can diffuse to the formation sites to grow nascent LDs. However two cases can be discriminated depending on the TG synthesis rate and mobility. In the case of an overall rapid synthesis rate (which is dependent on the synthesis enzymes expression levels and the synthesis reaction speed), nascent LDs are ignorant of each other, not necessarily communicating, because they are equally fed with TG molecules, grown, budded, and eventually detached. If instead TG diffusion is fast, the volume of nascent LDs will vary differently in time and their Laplace pressures will also change ([4] for review). In this situation, a TG flux can be established in the ER bilayer from very small droplets (at the pre-droplets stage to nascent LDs; large Laplace pressure) to bigger LDs (smaller Laplace pressure), Fig.4A. This phenomenon is called ripening and is responsible for the transfer of TG during adipocyte differentiation from smaller to bigger cytosolic LDs, and mediated by fat- specific protein p27[45]. Occurrence of ripening allows for the communication between forming LDs and can introduce variability on forming LD growth rate, size and number. Ripening is a slow process, driven by the differences in Laplace pressures and allowed by the diffusion of molecules from the volume of smaller droplets towards bigger droplets; sometimes it is confusingly referred to as fusion, which is a different process, featuring rapid surface and volume mixing of two droplets[4]. In fusion, two pre-droplets or nascent LDs could laterally approach and coalesce when close enough, Fig.4B. The fusion of two pre-droplets will increase the resulting pre-droplet volume that can become a nascent LD, i.e. $V>V^{*}$. Nascent LDs can fuse with pre-droplets, or with other nascent LDs, to form bigger LDs. Fusion events can rapidly generate more thermodynamically stable nascent LDs. Finally, both fusion and ripening could generate supersized LDs in the ER.

Proteins can potentially regulate ripening and fusion within the ER membrane. FIT proteins can bind TG molecules[34] and could modulate communication between LD sites by shuttling TG to 
specific sites; Seipin, present on LD formation sites[38, 39, 46], could stabilize nascent LDs and prevent their disappearance by preventing TG leakage; Seipin could also behave as a physical barrier that prevent lateral fusion between nascent LDs; finally, proteins such as ASCL3 or Perilipins can physically modulate the Laplace pressure of forming LDs, preventing small nascent LDs $\left(V\right.$ barely over $\left.V^{*}\right)$ from shrinking and mature LDs $\left(V>V^{*}\right)$ from growing faster. The exact role of proteins in modulating LD ripening or fusion in the ER is not known to date. Nonetheless, the potential actions listed above of the proteins would result in the regulation of the distribution of TG in forming LDs, by inhibiting or reinforcing communication between nascent LDs or pre-droplets. These potential functions are consistent with the frequently observed number and size variations of formed LDs when the proteins are knocked down[11, 32, 35, 47].

\section{LD budding}

Accumulating TG molecules is necessary but not sufficient to form a LD. A TG phase could demix but simply form a film or a flat lens between the two ER monolayers, growing mainly in a planar direction. LD biogenesis requires that the oil phase forms a nearly spherical droplet enclosed in a spherical protrusion of one of the ER monolayers, protrusion that eventually undergoes fission from the parent membrane to release the LD. The membrane deformation process prior to LD release is called budding.

\section{1) Budding mechanisms in biology and LD formation}

Membrane budding is ubiquitous in biology, especially in vesicular trafficking. The biogenesis of bilayer vesicles from the plasma, Golgi or ER membranes are often carried out by coat protein machineries, respectively Clathrin, complex protein I and II (COPI and COPII), which assemble on the membranes and polymerize to form a spherical shell enclosing the budded vesicles[14]. The size of these vesicles is generally in the range of $100 \mathrm{~nm}$ [14]. For LD biogenesis, with a size a priori superior to this value[32], budding is not known to be mediated by similar protein machineries; in such eventuality, COPI would be a good candidate. Among the known coat machineries, COPI was mainly shown to be important for LD formation and functionality[29, 48-51]. COPI is able to bud $60 \mathrm{~nm}$ nano-LD from artificial and purified LDs[50, 51]; whether it can assemble on specific sites of the ER to locally bud nano-LDs for LD formation is not known and cannot be excluded. Other protein candidates for LDs budding can be speculatively proteins having the ability to deform membranes; these proteins include caveolin-related proteins[52], or oleosins in plants. Caveolins deform bilayer membranes to form vesicles; they localize to LDs, 
especially under cholesterol rich conditions in adipocytes[21, 53], and could therefore physically contribute to the budding of LDs. Likewise, oleosins, crucial for the good packaging of plant lipid bodies (i.e. LDs) [54], could also physically contribute to budding [55]. Both protein types display hairpin motifs and a high positive curvature, and could naturally induce the ER outer monolayer deformation for budding [56, 57]. Many other LD proteins (e.g. DGAT2, Glycerol-3-phosphate Acyltransferase Isoform-4, Human Lysophosphatidylcholine Acyltransferases 1 and 2) [58-60] display hairpin motifs that can potentially induce LD budding. Finally, similarly to apolipoproteins that are able to bud tiny TG particles by binding through amphipathic helices to the ER luminal monolayer[61, 62], proteins binding LDs with amphiphathic helices to LDs can have a natural curvature that imposes the budding shape of LDs[33, 63]. Among them are Perilipins, major LD components[43].

However, the knockdown of most LD proteins does not prevent LD formation[29, 48]. Although, one cannot exclude the existence of yet unknown and redundant protein machineries mediating LD budding, this observation rather suggest that proteins essentially have a regulatory role such as controlling LD nucleation site[7] (Fig.2), or size[44], or protein content[29, 48, 50]; other factors may be consequently more deterministic for LD budding. While lack of protein involvement is uncommon in biology, the topology of LDs is also uncommon[4]: all other cellular organelles are bilayer-bounded, but LDs are bounded by a PL monolayer covering an oil phase. In the next section, we propose that the molecular interactions between oil molecules and PLs of the ER membrane are, in addition to nucleation, able to mediate LD budding without any energy input from proteins. In this picture, we will then discuss the possible role of proteins in the regulation of budding.

\section{2) From droplet dewetting to LD budding}

During LD biogenesis, a cytosolic and luminal ER PL monolayers cover TG molecules forming an oil lens of few tens of $\mathrm{nm}$ in diameter. This situation can be envisioned as three-phase wetting phenomenon[64], box 2. The three phases are in this case the cytosol, the ER lumen and the oil phase. The shape of the nascent LD should thus depend to a large extent on the surface tension of the interfaces, or membranes, separating the different phases, i.e. the surface energy cost for generating an interface between two fluids ([4] for review on surface tension). The surface tension of the ER bilayer membrane has been determined around $\gamma_{c l} \approx 10^{-2} \mathrm{mN} / \mathrm{m}[65]$. The surface tensions of the TG-cytosolic and TG-ER luminal monolayers are probably similar and can be expected to be of the order of $\gamma_{o l} \approx \gamma_{o c} \approx 0.1-1 \mathrm{mN} / \mathrm{m}$, which is the surface tension of 
reconstituted LD monolayer surface fully covered by PLs[51, 66]. These surface tension values are mainly determined by the PL composition of the different membranes and the oil phase[4].

LD budding to the cytosol corresponds to a partial or complete dewetting of the oil phase from the ER luminal PL monolayer (Fig.5A(3,4)), and could in principle be solely driven by the interfacial forces (i.e. originating from surface tension). By analogy with wetting droplets[67], box 2 and Fig.5A, the shape of a forming LD (Fig.5B) can be characterized by the contact angle $\alpha$, determined (at least in part) by the surface tensions $[67,68]$. Small values of the contact angle $\alpha$, i.e. close to $0^{\circ}(\mathrm{Fig} .5 \mathrm{~A}(3,4))$, correspond to budding.

The value of the angle has other important biological consequences besides budding. Small angles reflect the propensity of LDs to remain connected with the $\mathrm{ER}$, and thereby the capacity of proteins to reach them from the latter[50, 51, 59, 69]. For large contact angles, LD growth would be mainly by spreading; budding would eventually occur at larger size due to other constraints arising from ER topology or from proteins that could limit or prevent spreading. In cells, although LDs can appear in most cases perfectly spherical, they could be in a non-dewetting state; measuring the real contact angle in this context is challenging.

Proteins especially implicated in LD formation could influence the value of the contact angle to favor budding by modulating the different tensions. Seipin could localize to LD formation sites and modulate membrane tension, e.g. by locally mediating specific PL recruitment[38, 39, 70]. Other enzymes implicated in PL fabrication or modification could also change membrane surface tension by fabricating specific PLs[60, 71-77]. Finally, the direct binding of proteins to nascent LDs, such as Perilipins or ASCLs[7, 32], could also vary the tension of the TG-cytosolic monolayer.

\section{3) Influence of membrane rigidity}

Surface tension mainly influences the shape of the LD bud, as discussed previously. However, at the nascent LD stage, the rigidity of the ER PL monolayers has a non-negligible effect: it opposes membrane bending and flattens the LD. As the latter grows, its monolayer curvature decreases, reducing thereupon the influence of rigidity. Due to this competition between membrane bending rigidity and surface tension, nascent LD shape may progressively evolve from a flat lens after nucleation, due to the monolayer rigidity, to a spherical shape at large size, dictated by surface tension[78]. This competition could explain the existence of a typical LD budding size.

\section{4) Directional budding and the possible roles of proteins}


The origin of the directional budding of LDs, mainly occurring towards the cytosol, remains poorly understood. From a biophysics standpoint, budding directionality requires an asymmetry between the physical properties of the ER monolayers, which should originate from the different protein and lipid composition of the two monolayers, or from a difference in pressure between the ER lumen and the cytosol.

An asymmetry of tension can lead to directional budding by dewetting, box 2 . If the tension of the cytosolic monolayer is lower than that of the luminal one, partial or complete dewetting of the oil phase from the luminal monolayer is favored (cases in Fig.5A(3,4) and Fig.5B(2,3)). This situation happens for example if there is an excess PL in the ER cytosolic monolayer compared to its luminal one. This imbalance could happen with phosphatidyl choline whose synthesis enzymes reside in the cytosol $[60,73,79]$, or in the nucleus[73, 75, 80], where LDs bud off[75, 81].

An asymmetry in the bending properties of the ER monolayers could also contribute to the budding directionality. An intrinsic positive curvature of the outer monolayer of the ER membrane would favor budding toward the cytosol[10] (or to the nucleus[81, 82]). The binding to nascent LDs of cytosolic proteins with positive curvature[40] (e.g. by the insertion of amphipathic helix, or by the presence of bar-like domain), or an excess of PLs having a positive curvature in the ER cytosolic monolayer (e.g. Lyso-PLs), would favor bending of the ER monolayer and budding into the cytosol[83-85]. Transmembrane proteins could also generate positive curvature. Recent electron microscopy imaging suggest for example the implication of FIT1 in LD directional budding, as its deletion caused the ER luminal localization of LDs [36]. FIT1 proteins have indeed transmembrane multi-spanning domains and its $\mathrm{N}$ and $\mathrm{C}$ termini sitting in the cytosol, and therefore could predeform the ER cytosolic monolayer. Other proteins involved in TG synthesis or in LD formation can have similar effects. Finally, LD budding in the cytosol could arise from a protein crowding mechanism due to a protein concentration imbalance between each side of the ER membrane[85, 86]. Peripheral proteins bound to a bilayer or a monolayer membrane diffuse, collide and repel[80, 87], generating thereby a surface pressure. This pressure induces membrane curvature resulting for example to the appearance of bilayer protrusions toward the side of higher protein concentration[ 87,88$]$. Since nascent LDs are a priori asymmetrically bound by proteins, as most of their proteins bind from the cytosol, a spontaneous curvature of LD towards the cytosolic side could be favored by such crowding effect. 
Directional budding could also arise from ER topology. The ER has a small luminal volume and a high concentration of protein building blocks, which cause an excess pressure that may limit luminal budding by "pushing" forming LD toward the cytosol.

As a remark, all these mechanisms of membrane asymmetry could be involved in LD budding in the nucleus[81, 82].

Finally, in the particular case of lipoprotein (which can be seen as a particular type of LD), budding occurs towards the lumen. Since cytosolic budding is favored under normal circumstances, an energy input is required for inducing lipoprotein formation. This energy is possibly provided by microsomal triglyceride transfer proteins and/or apolipoproteins. Apolipoprotein bind with nanodisks-forming amphipathic helices that induce membrane curvature[61, 62]. By their association with the ER luminal monolayer[89], they could locally impose a curvature to the luminal monolayer, compensating the effect of asymmetry factors mediating cytosolic budding.

\section{Box 2: Wetting basic principles}

The equilibrium shape of a fluid drop (phase "o") at the interface between two liquids (phase "c" and "l") is determined by the surface tension - the interfacial energy per unit area [4] - of the three interfaces, $\gamma_{o c}, \gamma_{o l}$, and $\gamma_{c l}$. These tensions are determined by the molecular interactions between the components of the phases in contact[4, 15,67,68]. The propensity of the drop to spread along the interface, or to dewet, can be described using the tension-dependent parameters defined in Fig.5, $S=\gamma_{c l}-\gamma_{o c}-\gamma_{o l}$ (called spreading parameter) and $E=\gamma_{c l}+\gamma_{o c}-\gamma_{o l}$ (empirically called the entering parameter, being here the budded shape). Three situations can be met: total wetting (Fig.5A1), partial wetting (Fig.5A(2,3)) and dewetting (Fig.5A4)[4, 67, 68]. In the case of partial wetting, the shape of the drop is essentially characterized by the contact angle $\alpha$ in Fig.5, defined by the different surface tensions:

$$
\cos \alpha=\frac{\gamma_{o l}^{2}-\gamma_{c l}^{2}-\gamma_{o c}^{2}}{2 \gamma_{c l} \gamma_{o c}}
$$

For large values of $\alpha$, the drop forms a flattened lens (Fig.5B); when $\alpha$ is small, i.e. close to $0^{\circ}$, the drop is nearly spherical (Fig.5A(3,4)).

\section{Conclusion}


LDs, coated by a PL monolayer, can have a much higher budding energy as compared to bilayer vesicles[66]; it is hence plausible that cells develop/use alternative mechanisms for LD budding compared to the generation of bilayer vesicles. This review offers new orientations for studying LD formation. It can serve as a guideline for better studying LD formation and subsequent protein trafficking. Although speculative from the biology background, all the physics ingredients described, based on nucleation and dewetting phenomena, are relevant for LD biogenesis. We highlight overlooked ER physical chemistry properties, especially the interaction between lipids, which are probably fundamental for LD formation. In vitro experiments based on emulsion droplets should be of particular interest, as they allow a precise estimation of the contribution of lipids and proteins. What is the contribution of PLs in LD formation? Are there specific PLs facilitating LD nucleation? What is the contribution of dewetting in LD biogenesis? Do proteins regulate LD nucleation or does nucleation drive instead protein recruitment to nascent LDs? Among the proposed physical mechanisms resulting in membrane asymmetry, which ones are relevant for the cytosolic budding of LDs? Using a combination of in vitro and cell biology approaches will enable addressing these important questions of LD biology raised by the review.

\section{Acknowledgments}

Authors gratefully thank Dr. Luca Monticelli and Steve Donaldson for the critical read of the manuscript. This work was supported by the ATIP-Avenir program and the Programme Emergence de la ville de Paris to A.R.T. 
1. Walther, T.C. and R.V. Farese, Jr., Lipid droplets and cellular lipid metabolism. Annu Rev Biochem, 2012. 81: p. 687-714.

2. Martin, S. and R.G. Parton, Lipid droplets: a unified view of a dynamic organelle. Nature Reviews Molecular Cell Biology, 2006. 7(5): p. 373-378.

3. Thiele, C. and J. Spandl, Cell biology of lipid droplets. Curr Opin Cell Biol, 2008. 20(4): p. 378-85.

4. Thiam, A.R., R.V. Farese, and T.C. Walther, The Biophysics and Cell Biology of Lipid Droplets. Nature Reviews Molecular Cell Biology, 2013.

5. Welte, M.A., Expanding Roles for Lipid Droplets. Current Biology, 2015. 25(11): p. R470R481.

6. Wilfling, F., et al., Lipid droplet biogenesis. Curr Opin Cell Biol, 2014. 29: p. 39-45.

7. Pol, A., S.P. Gross, and R.G. Parton, Review: biogenesis of the multifunctional lipid droplet: lipids, proteins, and sites. J Cell Biol, 2014. 204(5): p. 635-46.

8. Ohsaki, Y., et al., Biogenesis of cytoplasmic lipid droplets: from the lipid ester globule in the membrane to the visible structure. Biochim Biophys Acta, 2009. 1791(6): p. 399-407.

9. Brasaemle, D.L. and N.E. Wolins, Packaging of fat: an evolving model of lipid droplet assembly and expansion. J Biol Chem, 2012. 287(4): p. 2273-9.

10. Sturley, S.L. and M.M. Hussain, Lipid droplet formation on opposing sides of the endoplasmic reticulum. J Lipid Res, 2012. 53(9): p. 1800-10.

11. Yang, H., et al., Controlling the size of lipid droplets: lipid and protein factors. Curr Opin Cell Biol, 2012. 24(4): p. 509-16.

12. Penno, A., G. Hackenbroich, and C. Thiele, Phospholipids and lipid droplets. Biochim Biophys Acta, 2013. 1831(3): p. 589-94.

13. McMahon, H.T. and J.L. Gallop, Membrane curvature and mechanisms of dynamic cell membrane remodelling. Nature, 2005. 438(7068): p. 590-6.

14. Hurley, J.H., et al., Membrane budding. Cell, 2010. 143(6): p. 875-87.

15. De Gennes, P.-G., F. Brochard-Wyart, and D. Quéré, Capillarity and wetting phenomena: drops, bubbles, pearls, waves. 2004: Springer.

16. Cases, S., et al., Identification of a gene encoding an acyl CoA : diacylglycerol acyltransferase, a key enzyme in triacylglycerol synthesis. Proceedings of the National Academy of Sciences of the United States of America, 1998. 95(22): p. 13018-13023.

17. Coleman, R.A. and D.P. Lee, Enzymes of triacylglycerol synthesis and their regulation. Progress in Lipid Research, 2004. 43(2): p. 134-176.

18. Yen, C.L.E., et al., DGAT enzymes and triacylglycerol biosynthesis. Journal of Lipid Research, 2008. 49(11): p. 2283-2301.

19. Turkish, A. and S.L. Sturley, Regulation of triglyceride metabolism. I. Eukaryotic neutral lipid synthesis: "Many ways to skin ACAT or a DGAT". American Journal of PhysiologyGastrointestinal and Liver Physiology, 2007. 292(4): p. G953-G957.

20. Buhman, K.K., H.C. Chen, and R.V. Farese, The enzymes of neutral lipid synthesis. Journal of Biological Chemistry, 2001. 276(44): p. 40369-40372.

21. Fujimoto, T. and R.G. Parton, Not just fat: the structure and function of the lipid droplet. Cold Spring Harb Perspect Biol, 2011. 3(3).

22. Barrat, J.-L. and J.-P. Hansen, Basic concepts for simple and complex liquids. 2003: Cambridge University Press.

23. Chandler, D., Interfaces and the driving force of hydrophobic assembly. Nature, 2005. 437(7059): p. 640-647.

24. Safran, S.A., Statistical thermodynamics of surfaces, interfaces, and membranes. Vol. 90. 1994: Perseus Books. 
25. Khandelia, H., et al., Triglyceride blisters in lipid bilayers: implications for lipid droplet biogenesis and the mobile lipid signal in cancer cell membranes. PLoS One, 2010. 5(9): p. e12811.

26. Hsieh, K., et al., Perilipin family members preferentially sequester to either triacylglycerol-specific or cholesteryl-ester-specific intracellular lipid storage droplets. J Cell Sci, 2012. 125(Pt 17): p. 4067-76.

27. Zanghellini, J., F. Wodlei, and H.H. von Grunberg, Phospholipid demixing and the birth of a lipid droplet. J Theor Biol, 2010. 264(3): p. 952-61.

28. Spanova, M., et al., Effect of lipid particle biogenesis on the subcellular distribution of squalene in the yeast Saccharomyces cerevisiae. J Biol Chem, 2010. 285(9): p. 612733.

29. Guo, Y., et al., Functional genomic screen reveals genes involved in lipid-droplet formation and utilization. Nature, 2008. 453(7195): p. 657-61.

30. Cohen, B.C., A. Shamay, and N. Argov-Argaman, Regulation of Lipid Droplet Size in Mammary Epithelial Cells by Remodeling of Membrane Lipid Composition-A Potential Mechanism. Plos One, 2015. 10(3).

31. Boutet, E., et al., Seipin deficiency alters fatty acid Delta 9 desaturation and lipid droplet formation in Berardinelli-Seip congenital lipodystrophy. Biochimie, 2009. 91(6): p. 796803.

32. Kassan, A., et al., Acyl-CoA synthetase 3 promotes lipid droplet biogenesis in ER microdomains. J Cell Biol, 2013. 203(6): p. 985-1001.

33. Robenek, H., et al., Adipophilin-enriched domains in the ER membrane are sites of lipid droplet biogenesis. Journal of Cell Science, 2006. 119(20): p. 4215-4224.

34. Gross, D.A., C.Y. Zhan, and D.L. Silver, Direct binding of triglyceride to fat storageinducing transmembrane proteins 1 and 2 is important for lipid droplet formation. Proceedings of the National Academy of Sciences of the United States of America, 2011. 108(49): p. 19581-19586.

35. Tan, J.S.Y., et al., Recent Advances in Understanding Proteins Involved in Lipid Droplet Formation, Growth and Fusion. Journal of Genetics and Genomics, 2014. 41(5): p. 251259.

36. Choudhary, V., et al., A conserved family of proteins facilitates nascent lipid droplet budding from the ER. J Cell Biol, 2015. 211(2): p. 261-71.

37. Skinner, J.R., et al., Diacylglycerol enrichment of endoplasmic reticulum or lipid droplets recruits perilipin 3/TIP47 during lipid storage and mobilization. J Biol Chem, 2009. 284(45): p. 30941-8.

38. Cartwright, B.R., et al., Seipin performs dissectible functions in promoting lipid droplet biogenesis and regulating droplet morphology. Mol Biol Cell, 2015. 26(4): p. 726-39.

39. Szymanski, K.M., et al., The lipodystrophy protein seipin is found at endoplasmic reticulum lipid droplet junctions and is important for droplet morphology. Proceedings of the National Academy of Sciences of the United States of America, 2007. 104(52): p. 20890-20895.

40. Shen, H., M. Pirruccello, and P. De Camilli, SnapShot: membrane curvature sensors and generators. Cell, 2012. 150(6): p. 1300-1300. e2.

41. Antonny, B., Mechanisms of membrane curvature sensing. Annu Rev Biochem, 2011. 80: p. 101-23.

42. Phillips, R., et al., Emerging roles for lipids in shaping membrane-protein function. Nature, 2009. 459(7245): p. 379-385.

43. Wolins, N.E., D.L. Brasaemle, and P.E. Bickel, A proposed model of fat packaging by exchangeable lipid droplet proteins. FEBS Lett, 2006. 580(23): p. 5484-91.

44. Suzuki, M., et al., Lipid droplets: size matters. J Electron Microsc (Tokyo), 2011.60 Suppl 1: p. S101-16. 
45. Gong, J., et al., Fsp27 promotes lipid droplet growth by lipid exchange and transfer at lipid droplet contact sites. J Cell Biol, 2011. 195(6): p. 953-63.

46. Fei, W.H., et al., Fld1p, a functional homologue of human seipin, regulates the size of lipid droplets in yeast. Journal of Cell Biology, 2008. 180(3): p. 473-482.

47. Brasaemle, D.L., Thematic review series: adipocyte biology. The perilipin family of structural lipid droplet proteins: stabilization of lipid droplets and control of lipolysis. J Lipid Res, 2007. 48(12): p. 2547-59.

48. Beller, M., et al., COPI complex is a regulator of lipid homeostasis. PLoS Biol, 2008. 6(11): p. e292.

49. Soni, K.G., et al., Coatomer-dependent protein delivery to lipid droplets. J Cell Sci, 2009. 122(Pt 11): p. 1834-41.

50. Wilfling, F., et al., Arf1/COPI machinery acts directly on lipid droplets and enables their connection to the ER for protein targeting. eLife, 2014. 3.

51. Thiam, A.R., et al., COPI buds 60-nm lipid droplets from reconstituted waterphospholipid-triacylglyceride interfaces, suggesting a tension clamp function. Proc Natl Acad Sci U S A, 2013. 110(33): p. 13244-9.

52. Cohen, A.W., et al., Role of caveolin-1 in the modulation of lipolysis and lipid droplet formation. Diabetes, 2004. 53(5): p. 1261-70.

53. Le Lay, S., et al., Cholesterol-induced caveolin targeting to lipid droplets in adipocytes: a role for caveolar endocytosis. Traffic, 2006. 7(5): p. 549-61.

54. Brown, D.A., Lipid droplets: proteins floating on a pool of fat. Curr Biol, 2001. 11(11): p. R446-9.

55. Murphy, D.J. and J. Vance, Mechanisms of lipid-body formation. Trends Biochem Sci, 1999. 24(3): p. 109-15.

56. Parton, R.G., M. Hanzal-Bayer, and J.F. Hancock, Biogenesis of caveolae: a structural model for caveolin-induced domain formation. J Cell Sci, 2006. 119(Pt 5): p. 787-96.

57. Ariotti, N., et al., Molecular Characterization of Caveolin-induced Membrane Curvature. J Biol Chem, 2015. 290(41): p. 24875-90.

58. Ohsaki, Y., M. Suzuki, and T. Fujimoto, Open questions in lipid droplet biology. Chem Biol, 2014. 21(1): p. 86-96.

59. Wilfling, F., et al., Triacylglycerol synthesis enzymes mediate lipid droplet growth by relocalizing from the ER to lipid droplets. Dev Cell, 2013. 24(4): p. 384-99.

60. Moessinger, C., et al., Human lysophosphatidylcholine acyltransferases 1 and 2 are located in lipid droplets where they catalyze the formation of phosphatidylcholine. J Biol Chem, 2011. 286(24): p. 21330-9.

61. Saito, H., S. Lund-Katz, and M.C. Phillips, Contributions of domain structure and lipid interaction to the functionality of exchangeable human apolipoproteins. Prog Lipid Res, 2004. 43(4): p. 350-80.

62. Mei, X. and D. Atkinson, Lipid-free apolipoprotein Al structure: insights into HDL formation and atherosclerosis development. Archives of medical research, 2015. 46(5): p. 351-360.

63. Rowe, E.R., et al., Conserved amphipathic helices mediate lipid droplet targeting of perilipins 1-3. J Biol Chem, 2016.

64. Kusumaatmaja, H. and R. Lipowsky, Droplet-induced budding transitions of membranes. Soft Matter, 2011. 7(15): p. 6914-6919.

65. Upadhyaya, A. and M.P. Sheetz, Tension in tubulovesicular networks of Golgi and endoplasmic reticulum membranes. Biophys J, 2004. 86(5): p. 2923-8.

66. Thiam, A.R. and F. Pincet, The Energy of COPI for Budding Membranes. Plos One, 2015. 10(7).

67. Bonn, D., et al., Wetting and spreading. Reviews of modern physics, 2009. 81(2): p. 739. 
68. Hotrum, N.E., et al., Monitoring entering and spreading of emulsion droplets at an expanding air/water interface: a novel technique. J Colloid Interface Sci, 2002. 247(1): p. 125-31.

69. Jacquier, N., et al., Lipid droplets are functionally connected to the endoplasmic reticulum in Saccharomyces cerevisiae. J Cell Sci, 2011. 124(Pt 14): p. 2424-37.

70. Grippa, A., et al., The seipin complex Fld1/Ldb16 stabilizes ER-lipid droplet contact sites. J Cell Biol, 2015. 211(4): p. 829-44.

71. Andersson, L., et al., PLD1 and ERK2 regulate cytosolic lipid droplet formation. J Cell Sci, 2006. 119(Pt 11): p. 2246-57.

72. Gubern, A., et al., Lipid droplet biogenesis induced by stress involves triacylglycerol synthesis that depends on group VIA phospholipase A2. J Biol Chem, 2009. 284(9): p. 5697-708.

73. Krahmer, N., et al., Phosphatidylcholine synthesis for lipid droplet expansion is mediated by localized activation of CTP:phosphocholine cytidylyltransferase. Cell Metab, 2011. 14(4): p. 504-15.

74. Shi, X., et al., Regulation of lipid droplet size and phospholipid composition by stearoylCoA desaturase. Journal of Lipid Research, 2013. 54(9): p. 2504-2514.

75. Aitchison, A.J., D.J. Arsenault, and N.D. Ridgway, Nuclear-localized CTP:phosphocholine cytidylyltransferase alpha regulates phosphatidylcholine synthesis required for lipid droplet biogenesis. Mol Biol Cell, 2015. 26(16): p. 2927-38.

76. Adeyo, O., et al., The yeast lipin orthologue Pah1p is important for biogenesis of lipid droplets. J Cell Biol, 2011. 192(6): p. 1043-55.

77. O'Hara, L., et al., Control of phospholipid synthesis by phosphorylation of the yeast lipin Pah1p/Smp2p Mg2+-dependent phosphatidate phosphatase. J Biol Chem, 2006. 281(45): p. 34537-48.

78. Foret, L., Shape and energy of a membrane bud induced by protein coats or viral protein assembly. European Physical Journal E, 2014. 37(5).

79. Moessinger, C., et al., Two different pathways of phosphatidylcholine synthesis, the Kennedy Pathway and the Lands Cycle, differentially regulate cellular triacylglycerol storage. Bmc Cell Biology, 2014. 15.

80. Kory, N., et al., Protein Crowding Is a Determinant of Lipid Droplet Protein Composition. Dev Cell, 2015. 34(3): p. 351-63.

81. Uzbekov, R. and P. Roingeard, Nuclear lipid droplets identified by electron microscopy of serial sections. BMC Res Notes, 2013. 6: p. 386.

82. Ohsaki, Y., et al., PML isoform II plays a critical role in nuclear lipid droplet formation. J Cell Biol, 2016. 212(1): p. 29-38.

83. McMahon, H.T. and J.L. Gallop, Membrane curvature and mechanisms of dynamic cell membrane remodelling. Nature, 2005. 438(7068): p. 590-596.

84. McMahon, H.T. and E. Boucrot, Membrane curvature at a glance. Journal of Cell Science, 2015. 128(6): p. 1065-1070.

85. Stachowiak, J.C., F.M. Brodsky, and E.A. Miller, A cost-benefit analysis of the physical mechanisms of membrane curvature. Nat Cell Biol, 2013. 15(9): p. 1019-27.

86. Derganc, J., B. Antonny, and A. Copic, Membrane bending: the power of protein imbalance. Trends Biochem Sci, 2013. 38(11): p. 576-84.

87. Stachowiak, J.C., et al., Membrane bending by protein-protein crowding. Nat Cell Biol, 2012. 14(9): p. 944-9.

88. Segrest, J.P., et al., Surface Density-Induced Pleating of a Lipid Monolayer Drives Nascent High-Density Lipoprotein Assembly. Structure, 2015. 23(7): p. 1214-26.

89. Ohsaki, Y., et al., Lipid droplets are arrested in the ER membrane by tight binding of lipidated apolipoprotein B-100. J Cell Sci, 2008. 121(Pt 14): p. 2415-22. 
Figure 1 TG demixing within the ER membrane. A) Triglyceride (TG) molecules can diffuse freely in the ER bilayer membrane. They interact with the bilayer components, in particular phospholipids (PL), the main ER component. B) TG molecules condensed in a LD mainly interact with other TGs. The energy gain from $A$ and $B$ mediates the spontaneous TG demixing and the formation of a TG lens in the bilayer.

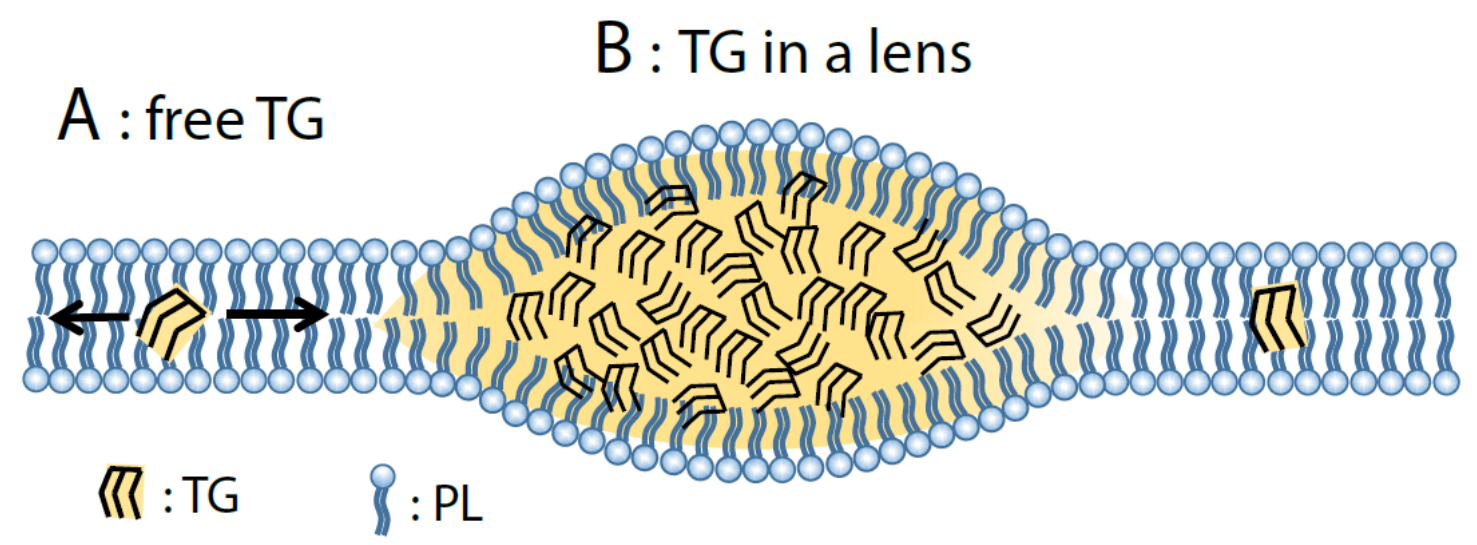

Figure 2 Different mechanisms can locally lower the LD nucleation energy. A) A local membrane deformation can favor LD nucleation. The case of a protrusion tip is shown. Accumulation of TG molecules at the tip of the protrusion is favored for nucleating a lens, because part of the energy required for deforming a monolayer during LD budding is already provided by the pre-deformation. Additionally, hydrophobic defects at the tip of the protrusion reflect a higher membrane tension than in the flat bilayer; therefore, accommodating TG molecules at the tip decreases the overall energy of the system. B) The local increase of the TG density near synthesis site will force the unzipping of the bilayer and the nucleation of a LD. C) The presence of curvature-inducing proteins, e.g. positively curved proteins, can decrease the energy cost for deforming the PL monolayer by TG accumulation. D) The presence of proteins and/or lipids interacting with TG, can decrease the nucleation energy by decreasing the interfacial energy of the droplet $\left(E_{S}\right.$ in box 1$)$ and favoring the condensation of TGs ( $\Delta \mu$ in box 1). 
Nucleation could be favored:

$A$ by membrane curvature

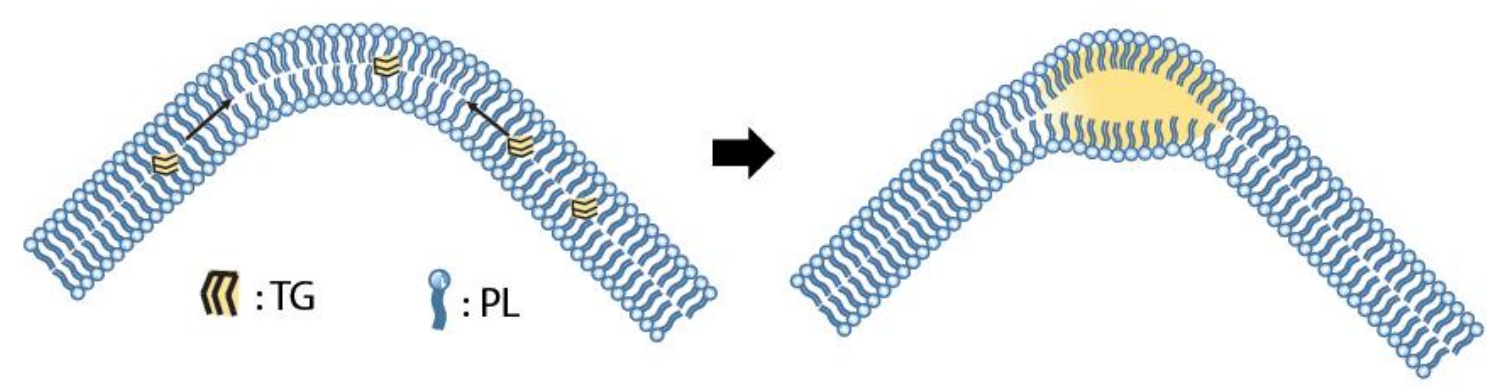

B at TG synthesis site

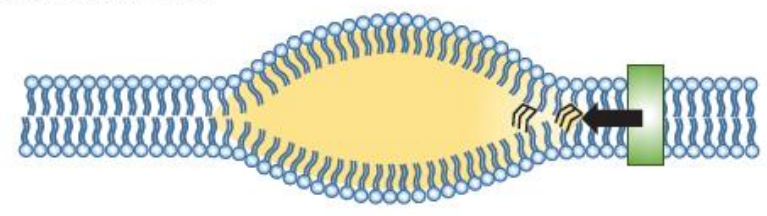

C by curvature-inducing proteins $(\nabla)$

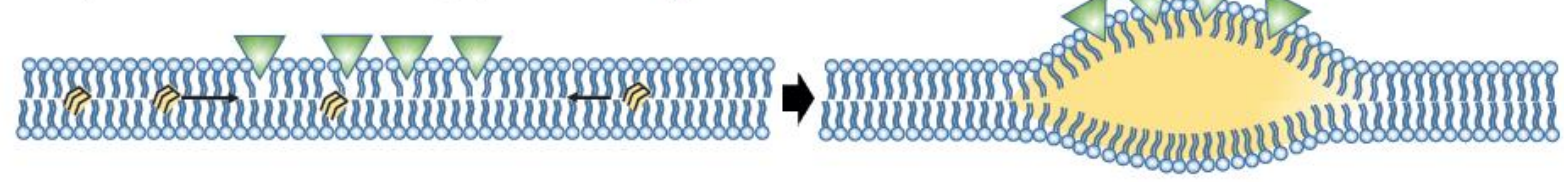

D by proteins/lipids $(00 \%)$ with favorable interaction with TG

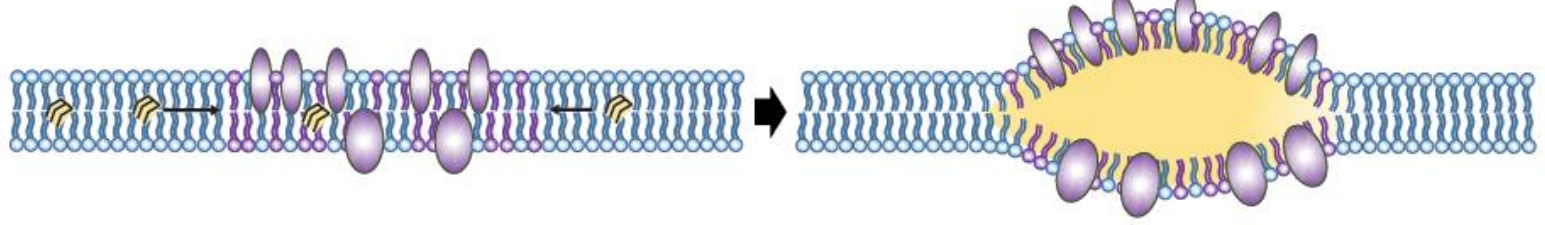

Figure 3 Nucleation of a LD induces a strong curvature or thickness increase of the membrane, and proteins can sense these physical membrane changes to bind nascent LD. 

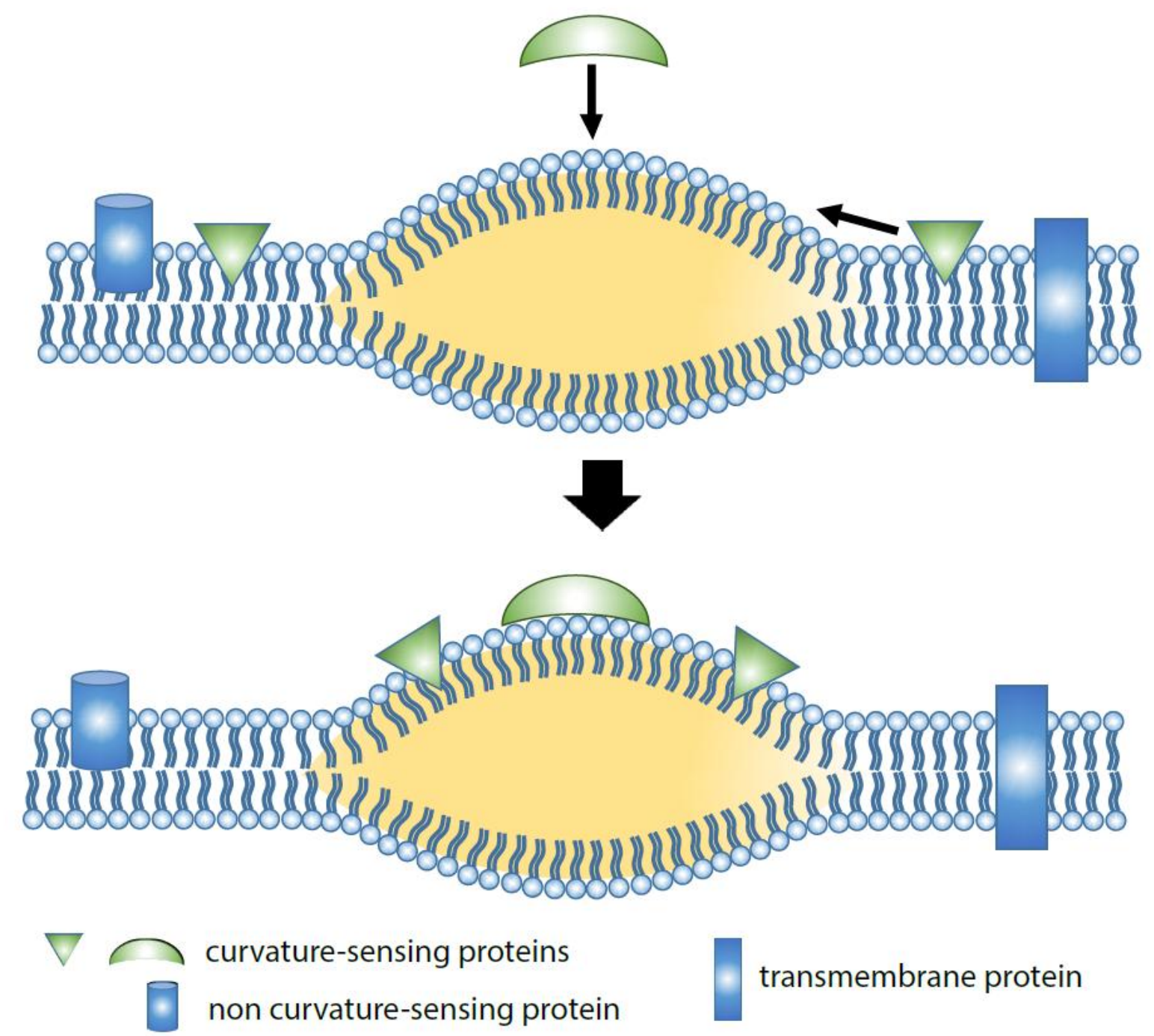

Figure 4 A) Droplet ripening: the difference of Laplace pressures can induce a flux of TG from small forming droplets (Pre-droplets or nascent LDs) to larger LDs within the ER bilayer. B) Predroplets and/or nascent LDs in the ER bilayer can fuse to former a bigger and more stable nascent LDs. 


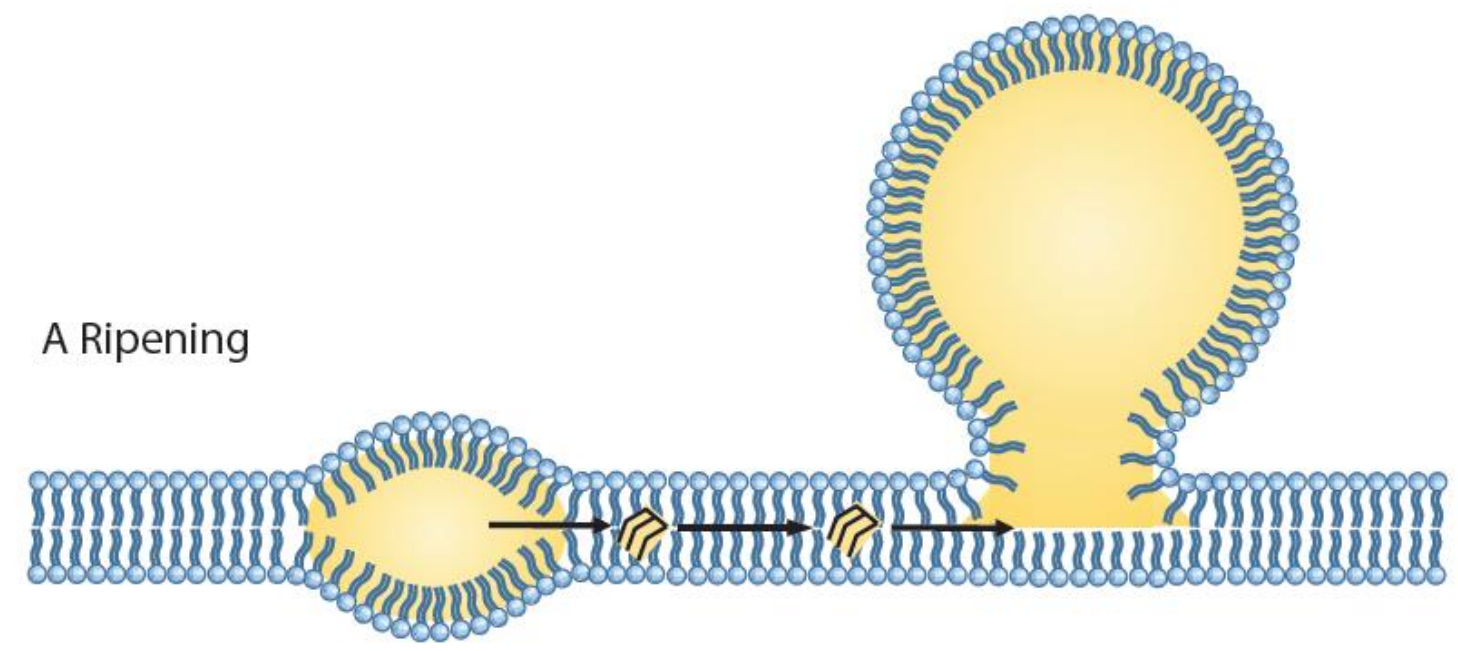

\section{B Fusion}
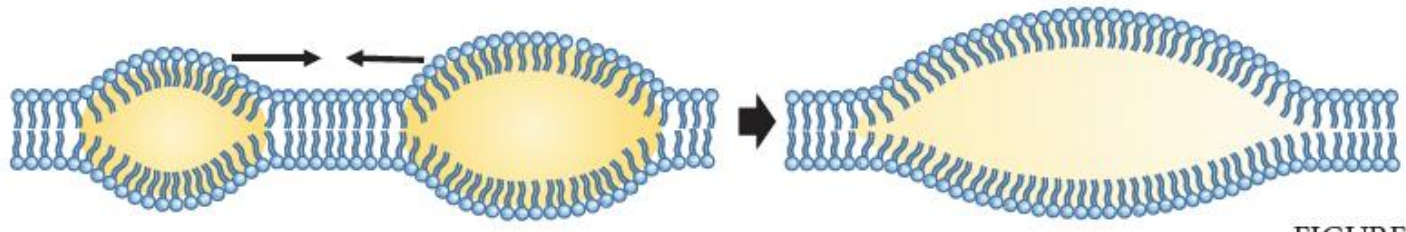

FIGURE 4

Figure $5 \mathrm{~A}$ ) Basics of a three fluid phases wetting situation. The shape of the yellow liquid droplet (o) in between two fluids (c) and (I) is determined by the tensions $\gamma_{o c}, \gamma_{o l}$ and $\gamma_{c l}$ of the interfaces between the different phases $0, \mathrm{l}$, and $\mathrm{c}$. Three cases can happen depending on the values of surface tensions: total wetting (1), partial wetting (2 and 3), and complete dewetting (4). In the case of partial wetting, the shape is characterized by the angle $\alpha$ determined by the value of the tensions: $\alpha$ is large in case 2 and small in case 3 where the droplet undergoes partial dewetting. B) A nascent LD can be considered as an oil phase in between the cytosol and the ER lumen. Following the physics of wetting phenomena, different shapes are possible in principle (B1 analogous to partial wetting $A 2, B 2$ analogous to partial dewetting $A 3$ and $B 3$ analogous to complete dewetting A4), depending on the tensions of the ER membrane and of the monolayers at the interfaces of the different phases. 
A
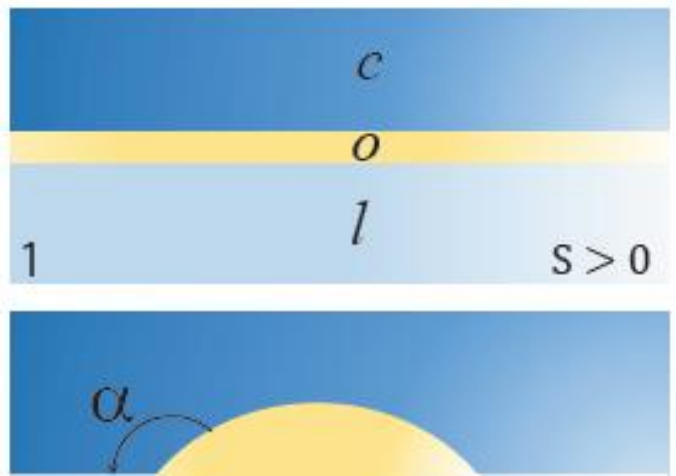

2

$\mathrm{S}<0<\mathrm{E}$

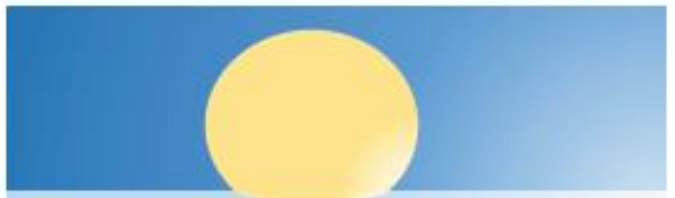

3

$\mathrm{S}<0<\mathrm{E}$
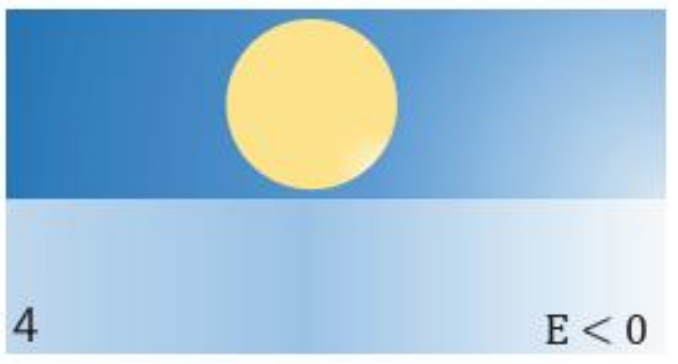

$\mathrm{S}=\gamma_{\mathrm{cl}}-\gamma_{\mathrm{oc}}-\gamma_{\mathrm{ol}} \quad \mathrm{E}=\gamma_{\mathrm{cl}}+\gamma_{\mathrm{oc}}-\gamma_{\mathrm{ol}}$
B
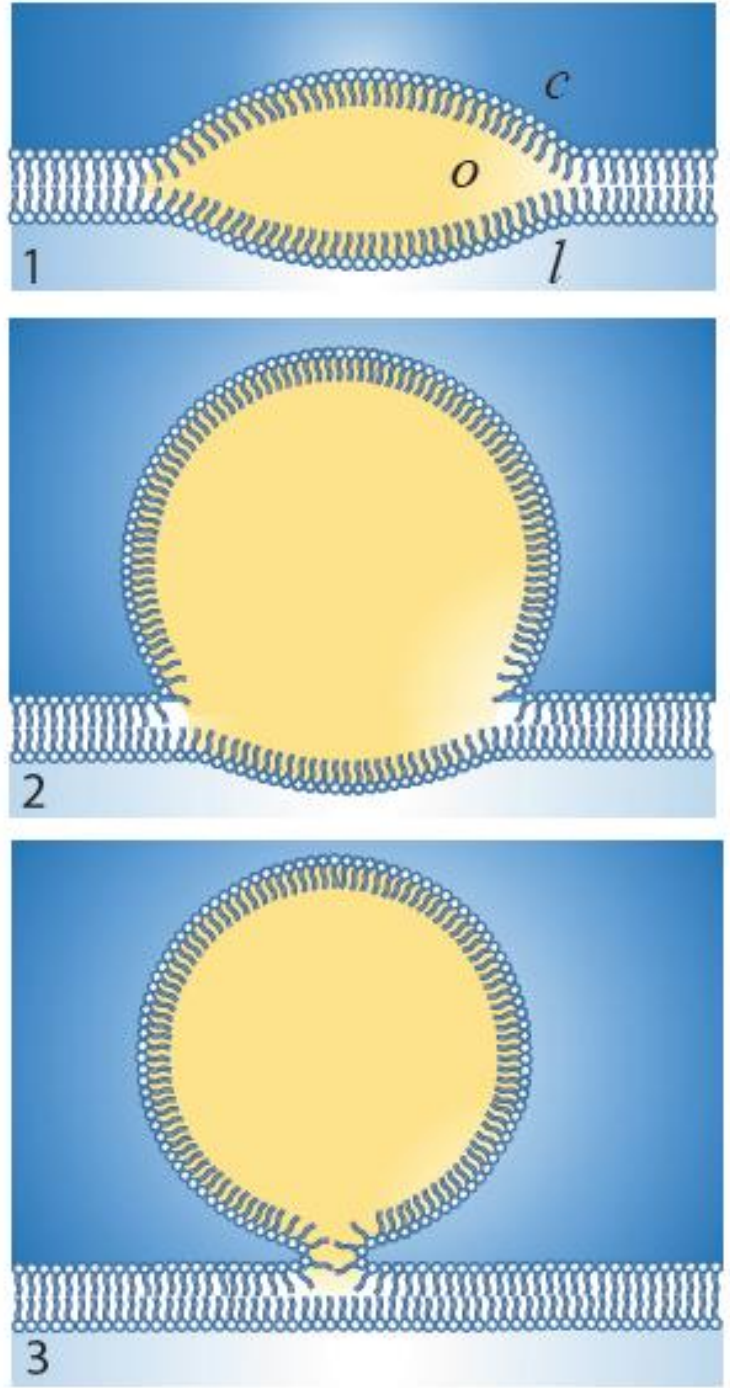
Box 1

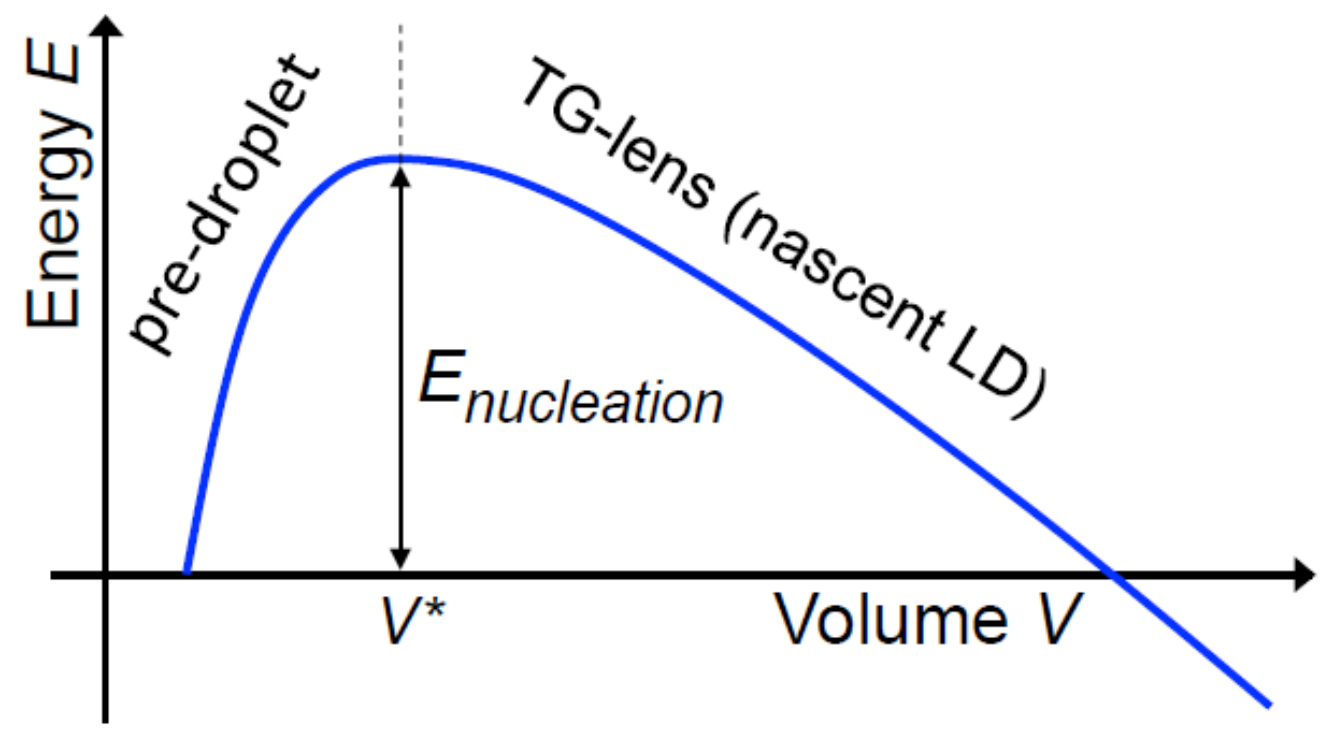

Table 1 Principal contributions (checked boxes) to the nucleation energy barrier of the different types of molecular interactions between Triglycerides (TG), Phospholipids (PL) and proteins, localized to LD formation sites. The nucleation energy has two contributions (see box 1): (i) the demixing free energy gained by the TG molecules forming a dense phase ( $\Delta \mu \rho V$ term in box 1) and (ii) the cost associated to the creation of the monolayer at the oil-water interface $\left(E_{S}\right.$ term in box 1). The later comprises two contributions: the monolayer interfacial energy, which depends on the molecular interactions between the monolayer components and TG, and the membrane deformation energy, which depends on the monolayer composition. 


\begin{tabular}{|c|c|c|c|}
\hline $\begin{array}{c}\text { Nucleation } \\
\text { energy }\end{array}$ & TG Demixing $(\Delta \mu)$ & \multicolumn{2}{|c|}{ Droplet interface $\left(E_{S}\right)$} \\
\cline { 3 - 4 } Interactions & & $\begin{array}{c}\text { Interfacial } \\
\text { energy }\end{array}$ & $\begin{array}{c}\text { Membrane } \\
\text { mechanics }\end{array}$ \\
\hline PL-TG & $\square$ & $\square$ & \\
\hline PL-PL & $\square$ & $\square$ & $\square$ \\
\hline TG-TG & $\square$ & $\square$ & \\
\hline Protein-TG & $\square$ & $\square$ & \\
\hline Protein-PL & & & $\square$ \\
\hline Protein-Protein & & & $\square$ \\
\hline
\end{tabular}

